\title{
Structural MRI markers of brain aging early after ischemic stroke OPEN
}

Emilio Werden, $\mathrm{PhD}$ Toby Cumming, $\mathrm{PhD}$ Qi Li, ME

Laura Bird, BSc Michele Veldsman, $\mathrm{PhD}$ Heath R. Pardoe, PhD Graeme Jackson, MD Geoffrey A. Donnan, MD Amy Brodtmann, MD, $\mathrm{PhD}$

Correspondence to Dr. Brodtmann: agbrod@unimelb.edu.au

Editorial, page 110

Supplemental data at Neurology.org

\section{ABSTRACT}

Objective: To examine associations between ischemic stroke, vascular risk factors, and MRI markers of brain aging.

Methods: Eighty-one patients (mean age $67.5 \pm 13.1$ years, 31 left-sided, 61 men) with confirmed first-ever $(n=66)$ or recurrent $(n=15)$ ischemic stroke underwent 3T MRI scanning within 6 weeks of symptom onset (mean $26 \pm 9$ days). Age-matched controls $(n=40)$ completed identical testing. Multivariate regression analyses examined associations between group membership and MRI markers of brain aging (cortical thickness, total brain volume, white matter hyperintensity [WMH] volume, hippocampal volume), normalized against intracranial volume, and the effects of vascular risk factors on these relationships.

Results: First-ever stroke was associated with smaller hippocampal volume $(p=0.025)$ and greater WMH volume $(p=0.004)$ relative to controls. Recurrent stroke was in turn associated with smaller hippocampal volume relative to both first-ever stroke $(p=0.017)$ and controls $(p=$ 0.001). These associations remained significant after adjustment for age, sex, education, and, in stroke patients, infarct volume. Total brain volume was not significantly smaller in first-ever stroke patients than in controls $(p=0.056)$, but the association became significant after further adjustment for atrial fibrillation ( $p=0.036$ ). Cortical thickness and brain volumes did not differ as a function of stroke type, infarct volume, or etiology.

Conclusions: Brain structure is likely to be compromised before ischemic stroke by vascular risk factors. Smaller hippocampal and total brain volumes and increased WMH load represent proxies for underlying vascular brain injury. Neurology ${ }^{\circledR}$ 2017;89:116-124

\section{GLOSSARY}

$\mathbf{A F}=$ atrial fibrillation; $\mathbf{B M I}=$ body mass index; CANVAS = Cognition and Neocortical Volume After Stroke; $\mathbf{C l}=$ confidence interval; ICV = intracranial volume; $\mathbf{S E}=$ standard error; TOAST = Trial of Org 10172 in Acute Stroke Treatment; T2DM = type II diabetes mellitus; $\mathbf{W M H}$ = white matter hyperintensity.

Ischemic stroke is associated with an increased risk of dementia, ${ }^{1}$ and the risk is even higher in recurrent stroke. ${ }^{2}$ The mechanisms underlying poststroke dementia are unclear. ${ }^{3}$ Macrovascular and microvascular disease, including cumulative microinfarct burden, may drive progressive cell loss and white matter degeneration. ${ }^{4}$ Cerebral infarcts are associated with secondary neurodegeneration in functionally and structurally connected brain regions, including areas distant to the primary lesion site. ${ }^{5-7}$ Vascular risk factors can also contribute to cerebral hypoperfusion and structural brain aging, ${ }^{8}$ with the hippocampi particularly vulnerable to vascular burden. ${ }^{7}$ Hippocampal atrophy is a cardinal feature of Alzheimer disease ${ }^{9}$ and is associated with dementia after stroke. ${ }^{10}$

The quantification of brain volume early after ischemic stroke allows the investigation of the effects on brain structure of both the stroke lesion and preexisting vascular risk factors. In the

\footnotetext{
From the Florey Institute of Neuroscience and Mental Health (E.W., T.C., Q.L., L.B., M.V., H.R.P., G.J., G.A.D., A.B.), University of Melbourne; Austin Health (G.J., A.B.), Heidelberg; and Eastern Clinical Research Unit (A.B.), Monash University, Box Hill Hospital, Melbourne, Victoria, Australia.

Go to Neurology.org for full disclosures. Funding information and disclosures deemed relevant by the authors, if any, are provided at the end of the article. The Article Processing Charge was funded by the authors.

This is an open access article distributed under the terms of the Creative Commons Attribution-NonCommercial-NoDerivatives License 4.0 (CC BY-NC-ND), which permits downloading and sharing the work provided it is properly cited. The work cannot be changed in any way or used commercially without permission from the journal.
} 
Cognition and Neocortical Volume After Stroke (CANVAS) study, ${ }^{11}$ we examine structural MRI markers of brain aging (hippocampal volume, total brain volume, cortical thickness, white matter hyperintensity $[\mathrm{WMH}])^{8}$ volume in prospectively recruited ischemic stroke patients scanned within 6 weeks after stroke (25.5 \pm 9.2 [7-42] days). We compare their results with results from age-matched controls who underwent identical scanning.

Given the deleterious effects of vascular burden and stroke on brain structure, we hypothesized that recurrent stroke patients would have smaller total and regional brain volumes than first-ever stroke patients and controls and that brain volumes would in turn be smaller in first-ever stroke patients than controls.

METHODS Standard protocol approvals, registrations, and patient consents. The study was approved by human research ethics committees of Austin Health, Eastern Health, and Melbourne Health. Informed consent was obtained from all participants.

Participants. The CANVAS study design and methodology have been described in detail previously. ${ }^{11}$ Briefly, patients with ischemic stroke, confirmed clinically and radiologically, were recruited within 6 weeks of their event from the acute stroke units at 3 hospitals in Melbourne, Australia (Austin Health, Box Hill Hospital, and Royal Melbourne Hospital). Patients with first-ever or recurrent ischemic stroke in any vascular territory and of any etiology according to Trial of Org 10172 in Acute Stroke Treatment $\left(\right.$ TOAST) ${ }^{12}$ and Oxfordshire ${ }^{13}$ classifications were included. Stroke patients were deemed ineligible if they received a diagnosis of TIA or primary hemorrhagic stroke, but ischemic stroke patients with secondary hemorrhagic transformation were included. Healthy control participants were selected from a larger database of volunteers who had previously undertaken MRI research at the Florey Institute of Neuroscience and Mental Health on the basis that they had no history of stroke or TIA and were of a comparable age, sex, and education to stroke patients. All participants had no history of dementia, neurodegenerative disorders, major psychiatric illnesses, or substance abuse problems.

Eighty-three stroke participants were scanned within 6 weeks of their ischemic stroke $(25.5 \pm 9.2[7-42]$ days after stroke). One participant was withdrawn from the study after extensive clinical investigations did not confirm ischemic stroke. MRI data for one stroke participant was excluded because of excessive movement. Eighty-one stroke patients had usable MRI data,

Table 1 Demographic, clinical, and vascular risk characteristics of the sample

\begin{tabular}{|c|c|c|c|c|c|c|c|c|c|}
\hline Demographic and clinical factors & \multicolumn{2}{|l|}{ RS } & \multicolumn{2}{|l|}{ FE } & \multicolumn{2}{|l|}{$\mathrm{HC}$} & \multicolumn{3}{|c|}{$p$ Value } \\
\hline Age, y, median (IQR) & 15 & $75(66-80)$ & 66 & $68(60.75-74)$ & 40 & $69(66-72)$ & $0.08^{a}$ & $0.55^{\mathrm{a}}$ & $0.07^{a}$ \\
\hline Male sex, n (\%) & 15 & 11 (73.3) & 66 & $50(75.8)$ & 40 & 25 (62.5) & $1.0^{\mathrm{b}}$ & $0.19^{b}$ & $0.54^{\mathrm{b}}$ \\
\hline Education, mean $\pm S D, y$ & 15 & $11.87 \pm 3.8$ & 66 & $12.90 \pm 3.88$ & 40 & $15.43 \pm 4.53$ & $0.33^{c}$ & $0.005^{c}$ & $0.009^{c}$ \\
\hline MoCA score, median (IQR) & 14 & $22(19-25.3)$ & 64 & $24(22-26.8)$ & 40 & $26.5(24-28)$ & 0.22 & $0.001^{a}$ & $0.004^{a}$ \\
\hline APOE $\varepsilon 4, n(\%)$ & 9 & 2 (22.2) & 50 & $10(20)$ & 24 & $2(8.3)$ & $1.0^{\mathrm{b}}$ & $0.32^{b}$ & $0.30^{\mathrm{b}}$ \\
\hline Married, n (\%) & 15 & $7(46.7)$ & 66 & 45 (68.2) & 40 & 25 (62.5) & $0.14^{\mathrm{b}}$ & $0.67^{b}$ & $0.36^{b}$ \\
\hline Depression, n (\%) & 15 & $3(20)$ & 66 & 7 (10.6) & 40 & $4(10)$ & $0.38^{\mathrm{b}}$ & $1.0^{\mathrm{b}}$ & $0.38^{\mathrm{b}}$ \\
\hline Stroke FH, n (\%) & 13 & $6(46.2)$ & 64 & $18(27.3)$ & 40 & $15(37.5)$ & $0.21^{\mathrm{b}}$ & $0.39^{b}$ & $0.75^{\mathrm{b}}$ \\
\hline High cholesterol, n (\%) & 15 & $9(60)$ & 66 & 27 (40.9) & 40 & $12(30)$ & $0.25^{\mathrm{b}}$ & $0.30^{\mathrm{b}}$ & $0.06^{b}$ \\
\hline T2DM, n (\%) & 15 & $6(40)$ & 66 & 14 (21.2) & 40 & $3(7.5)$ & $0.18^{\mathrm{b}}$ & $0.10^{\mathrm{b}}$ & $0.009^{b}$ \\
\hline$A F, n(\%)$ & 15 & $6(40)$ & 66 & $16(24.2)$ & 40 & $1(2.5)$ & $0.33^{b}$ & $0.002^{b}$ & $0.01^{\mathrm{b}}$ \\
\hline Smoking, pack-years, median (IQR) & 14 & $4(0-13)$ & 65 & $1(0-25)$ & 40 & $0(0-3)$ & $0.99^{a}$ & $0.05^{\mathrm{a}}$ & $0.15^{\mathrm{a}}$ \\
\hline $\mathrm{BMI} \geq 25 \mathrm{~kg} / \mathrm{m}^{2}, \mathrm{n}(\%)$ & 14 & 11 (73.3) & 66 & 53 (80.3) & 40 & $29(72.5)$ & $0.41^{\mathrm{b}}$ & $0.47^{\mathrm{b}}$ & $1.0^{\mathrm{b}}$ \\
\hline
\end{tabular}

Abbreviations: $\mathrm{AF}=$ atrial fibrillation; $\mathrm{BMI}=$ body mass index; $\mathrm{FE}=$ first-ever stroke; $\mathrm{FH}=$ family history; $\mathrm{HC}=$ healthy controls; IQCODE $=$ Informant Questionnaire on Cognitive Decline in the Elderly; IQR = interquartile range; MoCA = Montreal Cognitive Assessment; RS = recurrent stroke; T2DM = type Il diabetes mellitus.

a Mann-Whitney $U$ test.

${ }^{\mathrm{b}}$ Fisher exact test.

${ }^{\mathrm{c}}$ Independent-samples $t$ test. All tests were 2-tailed. 
including 66 patients with first-ever stroke and 15 patients with recurrent stroke. No patient had an old or acute stroke involving the hippocampus. Forty healthy controls were included.

Demographic, clinical, and vascular risk variables. We obtained information on age, years of education, handedness, marital status, stroke, and dementia family history via interview. The Montreal Cognitive Assessment was used as a measure of cognition and administered on the day of the MRI scan. We obtained an estimate of premorbid cognitive functioning with the Informant Questionnaire on Cognitive Decline in the Elderly (table 1).

We defined vascular risk factors as those modifiable and nonmodifiable factors that are associated with an increased risk of cerebrovascular disease, stroke, and dementia. Depression, hypertension, type II diabetes mellitus (T2DM), hypercholesterolemia and atrial fibrillation $(\mathrm{AF})$ were defined via a physician's diagnosis (reported by the participant) or via the use of medications for these conditions at the time of stroke or inclusion in the study (control participants). Smoking pack-years was defined as the number of cigarette packs smoked per day multiplied by the number of years the participant smoked. Body mass index (BMI) was calculated from weight and height measurements obtained on the day of the assessment and divided into low $(<25$ $\left.\mathrm{kg} / \mathrm{m}^{2}\right)$ and high $\left(\geq 25 \mathrm{~kg} / \mathrm{m}^{2}\right)$ groups.

Medical records were used to collect stroke information, including stroke side, thrombolysis treatment at the time of stroke, previous clinical stroke, and NIH Stroke Scale ${ }^{14}$ scores. Modified Rankin Scale (MRS) scores were obtained at the baseline assessment. ${ }^{15}$ An experienced stroke neurologist (A.B.) made TOAST $^{12}$ and Oxfordshire ${ }^{13}$ classifications. The final sample was heterogeneous with respect to stroke etiology and location (table e-1 at Neurology.org).

Blood samples were sent to the Centre for Translational Pathology, University of Melbourne for $A P O E$ genotyping, with carrier status determined by direct sequencing using methods described previously. ${ }^{16}$ APOE genotyping was optional.

MRI acquisition and processing. Participants were imaged on a 3T Siemens TIM Trio Scanner (Siemens, Munich, Germany) at the Melbourne Brain Centre, Austin Hospital campus of the Florey Institute of Neuroscience and Mental Health. MRIs were obtained with a T1-weighted 3-dimensional magnetization-prepared rapid gradient echo sequence with the following parameters: coronal slices with repetition time/echo time $=1,900$ milliseconds $/ 2.6$ milliseconds, TI $=900$ milliseconds, flip angle $=9^{\circ}$, slice thickness $=$ $1.0 \mathrm{~mm}$, matrix size $=256 \times 256$, number of slices $=160$, and voxel size $=1 \times 1 \times 1 \mathrm{~mm}^{3}$. A high-resolution, 3-dimensional sampling perfection with application-optimized contrasts using different flip angle evolutions (SPACE)-fluid-attenuated inversion recovery image was also acquired with 160 sagittal slices $1 \mathrm{~mm}$ thick, with 6,000-millisecond repetition time, 380-millisecond echo time, $120^{\circ}$ flip angle, and $256 \times 256$ acquisition matrix. T2 and diffusion-weighted images were acquired in the same imaging session. Total scan time was $\approx 25$ minutes. ${ }^{11,17}$

Images were processed with FreeSurfer version 5.1 with default processing settings used to obtain mean cortical thickness and total brain volume. Stroke infarcts were not masked before FreeSurfer analyses. To ensure that stroke infarcts did not distort tissue segmentations, FreeSurfer-reconstructed images were inspected, manually edited, and corrected if necessary before cortical thickness and brain volume estimates were calculated. Most segmentations did not require correction. Mean cortical thickness was defined as the average thickness of all cortical regions. Thirtyfive cortical regions per hemisphere are calculated by FreeSurfer. Values for these regions were averaged to produce mean cortical thickness. Total brain volume included gray matter, white matter, and cerebellum. Intracranial volume (ICV) was defined as gray matter, white matter, cerebellum, and CSF. Hippocampi were manually delineated by a structural imaging analyst (Q.L.) following European Alzheimer's Disease Consortium-Alzheimer's Disease Neuroimaging Initiative best-practice protocols. ${ }^{18,19}$ Manual segmentation of WMH on T2 fluid-attenuated inversion recovery images was performed in native space with Slicer 3D software (https://www.slicer.org/). The reproducibility of WMH manual masking was tested by 2 blinded raters (Q.L. and Fiona Permezel) on a random sample of 20 stroke and control participants (correlation $=0.90)$. Hippocampal and $\mathrm{WMH}$ volumes were calculated with FSL (http://fsl.fmrib.ox.ac.uk/fsl/fslwiki) ${ }^{20}$ after manual segmentation.

Statistical analyses. Data analyses were carried out with Stata release 13 (StataCorp, College Station, TX; 2013). Continuous clinical, demographic, and vascular risk data were compared between groups with independent-samples $t$ tests for normally distributed variables (means and SDs reported) and MannWhitney $U$ tests for skewed variables (medians and interquartile ranges reported). Fisher exact tests were used to compare $2 \times 2$ categorical data, and $\chi^{2}$ tests were used to compare categorical variables with $>2$ levels (frequencies and percentages reported). To correct for head size, each MRI variable (cortical thickness, total brain volume, WMH volume, hippocampal volume, and stroke infarct volume) was divided by the ICV to create a proportion. Hippocampal, WMH, and stroke infarct volumes were log-transformed after normalization against ICV because their distributions remained positively skewed. Corrected values were used in all regression analyses.

Robust regressions were used to examine associations between group membership and MRI variables. ${ }^{21}$ Three independent models were used. Comparisons between first-ever stroke patients and controls and between recurrent stroke patients and controls included age, sex, and education as covariates. Covariates in the recurrent and first-ever stroke comparisons included age, sex, education, and, to account for stroke size, normalized stroke infarct volume. Covariates were chosen a priori because they are known to affect brain volume. Vascular risk factors were added independently to these basic models to examine their effects on associations between group membership and MRI variables. Unstandardized regression (B) coefficients, standard errors (SEs), $p$ values, and $95 \%$ confidence intervals (CIs) were reported for all regression analyses. Levels of $\alpha$ were set at 0.05 (2-tailed). For all analyses, missing data were classified as missing at random and ignored.

RESULTS Eighty-one stroke patients (mean age 67.5 \pm 13.1 years, 31 left-sided, 61 men) and 40 control participants were included. Demographic, clinical, and vascular risk characteristics are presented in table 1. Stroke admission characteristics for the stroke groups are presented in table e-1. Raw values for cortical thickness, total brain volume, hippocampal volume, and WMH volume are presented in the figure. In the stroke group, there were no differences in regional brain volumes between the ipsilesional and contralesional hemispheres (table e-2). Thus, all data were averaged across hemispheres. Normalized cortical thickness and brain volumes did not differ according to stroke etiology or type $(p>0.05$ for all analyses of variance; data not shown). 

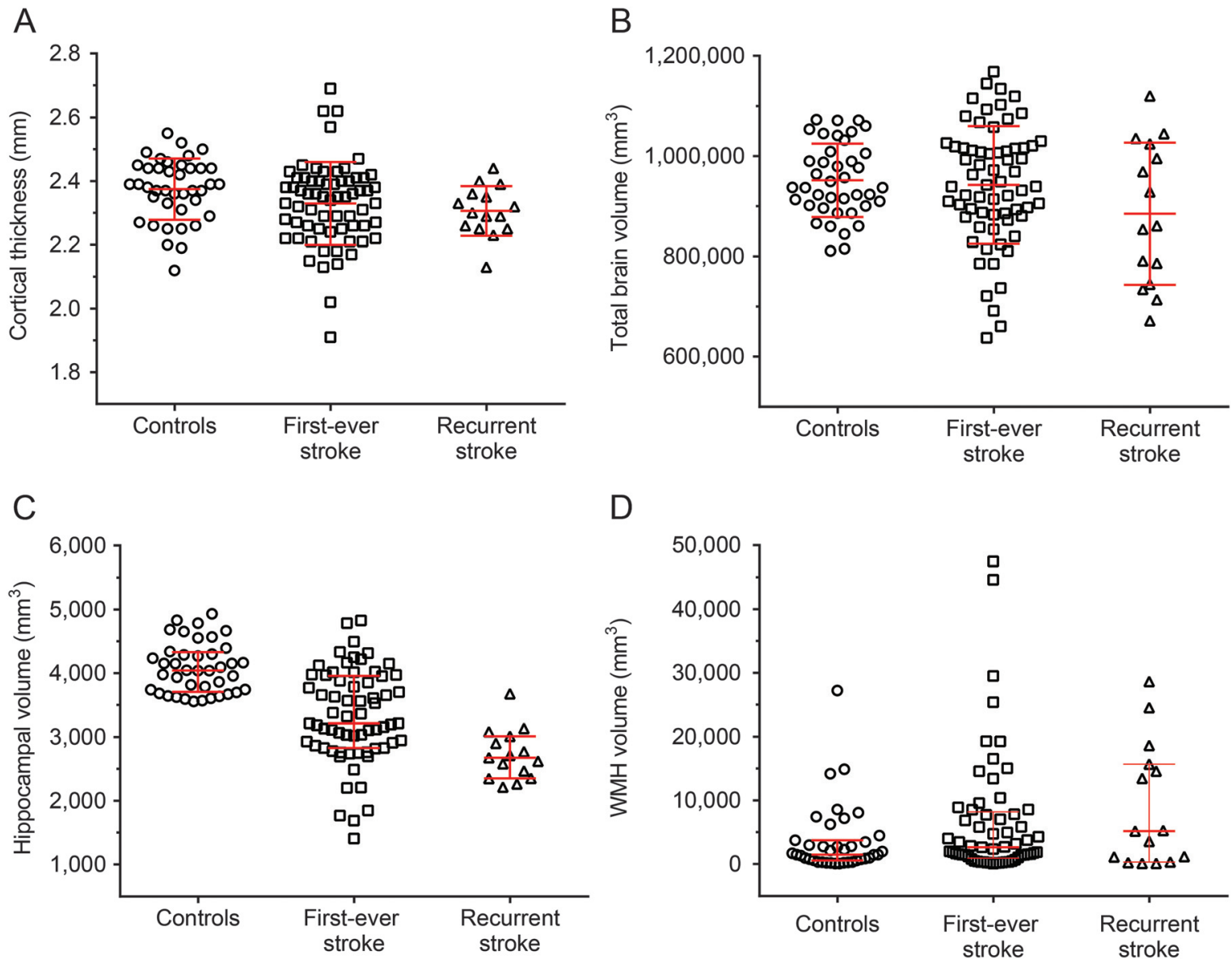

Values of cortical thickness (A), total brain volume (B), hippocampal volume (C), and white matter hyperintensity (WMH) volume (D) are presented for controls (circles), first-ever stroke patients (squares), and recurrent stroke patients (triangles). In panel A, values are expressed in millimeters. In panels B-D, values are expressed in millimeters cubed. The horizontal lines in panels A and B represent the mean values (middle) and SDs (bottom, top) for each group because raw scores were parametrically distributed. The horizontal lines in panels $C$ and $D$ represent median values (middle) and interquartile ranges (bottom, top) for each group because raw scores were nonparametrically distributed.

Demographic, clinical, and vascular risk data. Relative to healthy controls, first-ever and recurrent stroke patients performed worse on the Montreal Cognitive Assessment and were less educated, less likely to report a family history of dementia, and more likely to have $\mathrm{AF}$ (table 1). In addition, recurrent stroke patients were more likely than controls to have T2DM. Informant Questionnaire on Cognitive Decline in the Elderly scores were similar in all groups. Recurrent and first-ever stroke patients were comparable with respect to demographic and vascular risk factors (table 1). There was no significant difference in age between these 2 groups. Stroke admission characteristics were also similar between groups, but left-sided strokes were more common in the recurrent stroke group (table e-1).

First-ever stroke patients vs healthy controls. After adjustment for age, sex, and education, first-ever stroke was significantly associated with smaller loghippocampal volume and larger log-WMH volume but not with lower cortical thickness (table 2). The association between first-ever stroke and smaller total brain volume failed to reach statistical significance (table 2). First-ever stroke remained significantly associated with smaller log-hippocampal volume and larger log-WMH volume after the addition of each vascular risk factor to their respective models (tables e-3 and e-4).

Associations between first-ever stroke and cortical thickness remained nonsignificant with the addition of vascular risk factors; however, first-ever stroke became significantly associated with smaller total brain volume with the addition of AF to the model (table e-4).

No vascular risk factors were independently associated with log-hippocampal volume, total brain volume, or log-WMH volume (table e-4); however, a BMI $>25 \mathrm{~kg} / \mathrm{m}^{2}$ was associated with smaller cortical thickness regardless of group membership.

Recurrent vs first-ever stroke patients. Recurrent stroke was significantly associated with smaller log- 


\begin{tabular}{|c|c|c|c|c|c|}
\hline \multirow[t]{2}{*}{ Table 2} & \multicolumn{5}{|c|}{$\begin{array}{l}\text { Robust multivariate regressions for comparison of first-ever stroke and healthy controls, recurrent } \\
\text { stroke and first-ever stroke, and recurrent stroke and healthy controls }\end{array}$} \\
\hline & & B coefficient & SE & $p$ Value & $95 \% \mathrm{Cl}$ \\
\hline \multicolumn{6}{|c|}{ FE (1) vs HC (0) } \\
\hline \multicolumn{2}{|c|}{ Cortical thickness } & $-6.64 \times 10^{-8}$ & $9.99 \times 10^{-8}$ & 0.51 & $-2.7 \times 10^{-7}$ to $1.3 \times 10^{-7}$ \\
\hline \multicolumn{2}{|c|}{ Total brain volume } & -0.01 & 0.006 & 0.056 & -0.02 to $3 \times 10^{-4}$ \\
\hline \multicolumn{2}{|c|}{ Log-WMH volume } & 0.35 & 0.12 & 0.004 & 0.12 to 0.58 \\
\hline \multicolumn{2}{|c|}{ Log-hippocampal volume } & -0.02 & 0.008 & 0.025 & -0.03 to -0.002 \\
\hline \multicolumn{6}{|c|}{ RS (1) vs FE (0) } \\
\hline \multicolumn{2}{|c|}{ Cortical thickness } & $2 \times 10^{-7}$ & $1.5 \times 10^{-7}$ & 0.19 & $-9.9 \times 10^{-8}$ to $5 \times 10^{-7}$ \\
\hline \multicolumn{2}{|c|}{ Total brain volume } & 0.002 & 0.01 & 0.85 & -0.02 to 0.03 \\
\hline \multicolumn{2}{|c|}{ Log-WMH volume } & -0.11 & 0.18 & 0.56 & -0.47 to 0.26 \\
\hline \multicolumn{2}{|c|}{ Log-hippocampal volume } & -0.03 & 0.01 & 0.017 & -0.06 to -0.006 \\
\hline \multicolumn{6}{|c|}{ RS (1) vs HC (0) } \\
\hline \multicolumn{2}{|c|}{ Cortical thickness } & $4.8 \times 10^{-8}$ & $8.1 \times 10^{-8}$ & 0.56 & $-1.2 \times 10^{-7}$ to $2.1 \times 10^{-7}$ \\
\hline \multicolumn{2}{|c|}{ Total brain volume } & -0.005 & 0.005 & 0.31 & -0.02 to 0.005 \\
\hline \multicolumn{2}{|c|}{ Log-WMH volume } & 0.09 & 0.11 & 0.39 & -0.12 to 0.31 \\
\hline \multicolumn{2}{|c|}{ Log-hippocampal volume } & -0.02 & 0.006 & 0.001 & -0.03 to -0.009 \\
\hline
\end{tabular}

Abbreviations: $\mathrm{Cl}=$ confidence interval; $\mathrm{FE}=$ first-ever stroke; $\mathrm{HC}=$ healthy controls; $\mathrm{RS}=$ recurrent stroke; $\mathrm{SE}=$ standard error; $\mathrm{WMH}=$ white matter hyperintensity.

All MRI variables were normalized against intracranial volume before the analyses. Age, sex, and education were included as covariates in the FE and $\mathrm{HC}$ model and RS and $\mathrm{HC}$ model. Log-stroke infarct volume was included as an additional covariate in the RS and FE models. WMH could not be calculated for 5 FE participants and 1 RS participant. Stroke volume could not be calculated for 1 RS participant.

hippocampal volume but not with lower cortical thickness, smaller total brain volume, or lower logtransformed WMH volume after adjustments for age, sex, education, and log-stroke infarct volume (table 2).

Recurrent stroke remained significantly associated with smaller log-hippocampal volume after the addition of each vascular risk factor (table e-4). Associations between recurrent stroke and cortical thickness, total brain volume, and log-WMH volume remained nonsignificant (table e-4). No vascular risk factors were independently associated with any MRI variable (table e-4). Log-stroke infarct volume was not independently associated with any MRI variable in the basic models (data not shown).

Recurrent stroke patients vs healthy controls. Recurrent stroke was significantly associated with smaller loghippocampal volume after adjustments for age, sex, and education but not with cortical thickness, total brain volume, or log-transformed WMH volume (table 2).

The addition of vascular risk factors did not affect the results: recurrent stroke remained significantly associated with smaller log-hippocampal volume, and associations with total brain volume, cortical thickness, and log-WMH volume remained nonsignificant (table e-4).

No vascular risk factors were independently associated with log-hippocampal or total brain, volumes; however, T2DM was significantly associated with greater cortical thickness, AF was significantly associated with smaller log-WMH volume, and a BMI $>25 \mathrm{~kg} / \mathrm{m}^{2}$ was significantly associated with greater log-WMH volume (table e-4).

Associations of cortical thickness and brain volumes in stroke patients. Robust univariate regressions were conducted to determine whether cortical thickness and brain volumes were associated with stroke admission characteristics and APOE \&4 status (table 3). Smaller hippocampal and total brain volumes were associated with greater disability (Rankin Scale score) at the time of the assessment; however, after adjustment for age, sex, education, and log-stroke infarct volume, associations with hippocampal $(\mathrm{B}=-0.01$, $\mathrm{SE}=0.01, p=0.27,95 \% \mathrm{CI}=-0.04$ to 0.01$)$ and total brain $(\mathrm{B}=-0.001, \mathrm{SE}=0.001, p=0.94$, $95 \% \mathrm{CI}=-0.02$ to 0.02 ) volumes were no longer significant. Larger log-WMH volume was associated with smaller total brain volume (table 3 ). This association remained significant in the fully adjusted model $(\mathrm{B}=-0.02$, $\mathrm{SE}=0.01, p=0.045,95 \%$ $\mathrm{CI}=-0.03$ to $\left.3.6 \times 10^{-4}\right)$. No other associations were significant.

DISCUSSION In the first 6 weeks after stroke, we found smaller hippocampal and larger WMH volumes in first-ever stroke patients compared to 


\begin{tabular}{|c|c|c|c|c|}
\hline \multirow[t]{2}{*}{ Table 3} & \multirow[b]{2}{*}{ B coefficient } & \multicolumn{3}{|c|}{$\begin{array}{l}\text { amining associations between clinical and stroke admission } \\
\text { aging }\end{array}$} \\
\hline & & SE & $P$ Value & $95 \% \mathrm{Cl}$ \\
\hline \multicolumn{5}{|l|}{ Log-hippocampal volume } \\
\hline Left-sided stroke (1) & -0.006 & 0.01 & 0.56 & -0.03 to 0.02 \\
\hline tPA (1) & 0.005 & 0.01 & 0.73 & 0.02 to 0.03 \\
\hline Cortical stroke (1) & -0.008 & 0.01 & 0.52 & -0.03 to 0.02 \\
\hline Log-WMH volume/ICV & -0.01 & 0.01 & 0.23 & -0.02 to 0.006 \\
\hline Admission NIHSS & 0.002 & 0.002 & 0.25 & -0.001 to 0.005 \\
\hline Moderate/severe Rankin (1) & -0.02 & 0.01 & 0.04 & -0.04 to $-9.1 \times 10^{-4}$ \\
\hline APOE \&4 positive (1) & $2.3 \times 10^{-4}$ & 0.02 & 0.99 & -0.03 to 0.03 \\
\hline \multicolumn{5}{|l|}{ Total brain volume } \\
\hline Left-sided stroke (1) & -0.01 & 0.01 & 0.26 & -0.04 to 0.01 \\
\hline tPA (1) & 0.01 & 0.02 & 0.50 & -0.02 to 0.04 \\
\hline Cortical stroke (1) & -0.006 & 0.01 & 0.59 & -0.03 to 0.02 \\
\hline Log--WMH volume/ICV & -0.04 & 0.01 & $<0.001$ & -0.05 to -0.02 \\
\hline Admission NIHSS & -0.001 & 0.002 & 0.51 & -0.005 to 0.003 \\
\hline Moderate/severe Rankin (1) & -0.03 & 0.01 & 0.008 & -0.05 to -0.008 \\
\hline APOE \&4 positive (1) & 0.02 & 0.02 & 0.28 & -0.01 to 0.05 \\
\hline \multicolumn{5}{|l|}{ Cortical thickness } \\
\hline Left-sided stroke (1) & $-2.1 \times 10^{-7}$ & $2.2 \times 10^{-7}$ & 0.33 & $-6.4 \times 10^{-7}$ to $2.2 \times 10^{-7}$ \\
\hline tPA (1) & $1.4 \times 10^{-7}$ & $1.4 \times 10^{-7}$ & 0.34 & $-1.5 \times 10^{-7}$ to $4.2 \times 10^{-7}$ \\
\hline Cortical stroke (1) & $1.6 \times 10^{-8}$ & $1.3 \times 10^{-7}$ & 0.90 & $-2.4 \times 10^{-7}$ to $2.7 \times 10^{-7}$ \\
\hline Log--WMH volume/ICV & $-1.3 \times 10^{-7}$ & $1.2 \times 10^{-7}$ & 0.25 & $-3.7 \times 10^{-7}$ to $9.7 \times 10^{-8}$ \\
\hline Admission NIHSS & $1.2 \times 10^{-8}$ & $1.8 \times 10^{-8}$ & 0.51 & $-2.5 \times 10^{-8}$ to $4.9 \times 10^{-8}$ \\
\hline Moderate/severe Rankin (1) & $-1.2 \times 10^{-8}$ & $1.2 \times 10^{-7}$ & 0.92 & $-2.4 \times 10^{-7}$ to $2.2 \times 10^{-7}$ \\
\hline APOE $\varepsilon 4$ positive (1) & $2.4 \times 10^{-7}$ & $2.7 \times 10^{-7}$ & 0.38 & $-3 \times 10^{-7}$ to $7.7 \times 10^{-7}$ \\
\hline \multicolumn{5}{|l|}{ Log-WMH volume } \\
\hline Left-sided stroke (1) & 0.15 & 0.17 & 0.38 & -0.20 to 0.50 \\
\hline tPA (1) & -0.27 & 0.22 & 0.23 & -0.72 to 0.18 \\
\hline Cortical stroke (1) & -0.03 & 0.21 & 0.91 & -0.45 to 0.40 \\
\hline Admission NIHSS & 0.01 & 0.03 & 0.67 & -0.05 to 0.08 \\
\hline Moderate/severe Rankin (1) & 0.13 & 0.19 & 0.50 & -0.25 to 0.51 \\
\hline APOE $\varepsilon 4$ positive (1) & -0.13 & 0.24 & 0.59 & -0.60 to 0.35 \\
\hline
\end{tabular}

Abbreviations: $\mathrm{Cl}=$ confidence interval; ICV $=$ intracranial volume; NIHSS = NIH Stroke Scale; tPA = tissue plasminogen activator; $\mathrm{WMH}=$ white matter hyperintensity.

healthy, age-matched controls. Hippocampal volumes were smaller in recurrent stroke patients than in first-ever stroke patients and controls. Associations were significant after adjustments for demographic variables, vascular risk, and, in stroke patients, infarct volume. Total brain volume was significantly smaller in first-ever stroke patients relative to controls after adjustment for AF. Collectively, these results suggest that brain structure is compromised in first-ever stroke patients before their stroke, possibly by cumulative vascular disease. They also suggest that recurrent stroke may lead to accelerated hippocampal volume losses that are in addition to changes associated with exposure to vascular risk factors.

Our finding of further hippocampal volume loss in recurrent stroke patients may represent a "dosedependent" response to incident infarction. ${ }^{5}$ It is consistent with human and animal studies that have reported progressive hippocampal volume loss after ischemic stroke in extrahippocampal regions ${ }^{6,22}$ and suggests that stroke may be associated with remote damage to the hippocampus. ${ }^{6}$ Moreover, it suggests that our recurrent stroke patients are at risk of cognitive decline and dementia. ${ }^{23}$ 
It is possible that stroke triggered rapid hippocampal volume loss in our first-ever ischemic stroke patients, given the vulnerability of this structure to reductions in cerebral blood flow. ${ }^{7,24,25}$ In support of this notion, estimates of premorbid cognition were comparable between groups, but first-ever stroke patients performed worse than controls on a cognitive screening tool after stroke. However, it is unlikely that differences in hippocampal volume reported here between first-ever stroke patients and controls can be accounted for by poststroke change. We previously reported nonsignificant hippocampal volume loss in the first 3 months after stroke, comparing a 3-month scan with hyperacute scanning at 2 hours. ${ }^{26}$ In the animal literature, hippocampal neurodegeneration has been reported between 12 hours $^{27}$ and 150 days $^{22}$ after middle cerebral artery occlusion, but this is typically greater on the ipsilesional side. ${ }^{22}$ In the current study, hippocampal and brain volume losses were extensive and bilateral. Hippocampal volumes were also unrelated to infarct size and were not smaller in patients with posterior circulation strokes.

It is far more likely that smaller hippocampal volumes in our first-ever ischemic stroke patients represent the cumulative effect of vascular burden. In prior studies, patients have been scanned at different time points after stroke, producing conflicting results and making the effects of stroke and vascular risk factors on brain volumes difficult to separate. ${ }^{6,26,28,29}$ Our results suggest that brain volume loss and increased WMH burden are proxies for underlying vascular brain injury. ${ }^{30,31}$

The association between first-ever stroke and smaller total brain volume was significant only after adjustment for AF. This result is unsurprising given that exposure to vascular risk factors in mid and late life is known to affect brain structure, ${ }^{32-34}$ with $\mathrm{AF}$ particularly detrimental to hippocampal ${ }^{34}$ and wholebrain $^{35}$ volumes and white matter. ${ }^{36}$ The observed association between AF and reduced WMH load in the recurrent stroke and healthy control comparisons was unexpected, but we must note that this result is based on 7 participants with AF (6 recurrent stroke patients, 1 control). We confirmed an association between higher BMI and reduced cortical thickness ${ }^{37}$ and between higher BMI and greater WMH burden. ${ }^{38}$ The observed association of T2DM and greater cortical thickness may reflect a sample size or methodological issue: we examined mean cortical thickness across hemispheres, whereas prior studies have often reported region-specific cortical thinning in T2DM (e.g., right hemisphere, ${ }^{32}$ left anterior cingulate, ${ }^{39}$ temporal/parietal lobe ${ }^{40}$ ).

The strengths of the current study include the well-characterized stroke sample, the short interval between stroke and MRI scan, and the inclusion of a healthy, age-matched control group. Moreover, all participants were scanned on the same 3T MRI machine, and a single trained investigator completed all manual hippocampal and WMH tracings. The limitations of the study include the small stroke sample size, which meant that we could not examine MRI markers of brain aging in a more homogeneous sample. It also might have influenced the size of the B coefficients and CIs observed in some of our analyses. In addition, most participants in the stroke group experienced relatively minor strokes (i.e., NIH Stroke Scale scores were between 0 and 7 at admission), meaning the sample is representative of the mild to moderate stroke population only. Finally, the results must be interpreted with caution because the control sample size was small, and although we controlled for demographic factors (e.g., age, sex, and education) in all regression analyses, controls were better educated than stroke patients.

We found smaller brain volumes and increased WMH load in first-ever ischemic stroke patients. Recurrent stroke was associated with greater hippocampal volume loss. Our findings indicate that markers of brain aging are evident shortly after first stroke, with recurrent stroke having additive effects on the hippocampus.

\section{AUTHOR CONTRIBUTIONS}

Dr. Emilio Werden: writing of manuscript; data extraction, analysis, and interpretation; statistical analysis; preparation of figure and tables. Dr. Toby Cumming: study design and conceptualization; data interpretation; critical revision of manuscript. Qi Li and Laura Bird: data extraction; critical revision of manuscript. Dr. Michele Veldsman: critical revision of manuscript. Dr. Heath Pardoe: study design and conceptualization; critical revision of manuscript. Drs. Graeme Jackson and Geoffrey Donnan: study design and conceptualization. Dr. Amy Brodtmann: study design and conceptualization; data interpretation; critical revision of manuscript.

\section{ACKNOWLEDGMENT}

The authors thank the Victorian Life Sciences Computation Initiative at the University of Melbourne; Dr. Fiona Permezel, who kindly assisted with the hippocampal maskings; the MRI radiographers at Melbourne Brain Centre; and all our participants who generously contributed their time to the study.

\section{STUDY FUNDING}

This work was supported by the National Health and Medical Research Council project grant (APP1020526), Brain Foundation, Wicking Trust, Collie Trust, and Sidney and Fiona Myer Family Foundation. The Florey Institute of Neuroscience and Mental Health acknowledges the support received from the Victorian Government via the Operational Infrastructure Support Scheme.

\section{DISCLOSURE}

E. Werden reports no disclosures relevant to the manuscript. T. Cumming is on the editorial board of BMC Neurology. Q. Li, L. Bird, M. Veldsman, H. Pardoe, and G. Jackson report no disclosures relevant to the manuscript. G. Donnan is on the editorial board of International Journal of Stroke. A. Brodtmann is on the editorial boards of Neurology and International Journal of Stroke. Go to Neurology.org for full disclosures.

Received November 15, 2016. Accepted in final form March 16, 2017. 


\section{REFERENCES}

1. Pendlebury S, Rothwell P. Prevalence, incidence, and factors associated with pre-stroke and post-stroke dementia: a systematic review and meta-analysis. Lancet Neurol 2009;8:1006-1018.

2. Desmond DW, Moroney JT, Paik MC, et al. Frequency and clinical determinants of dementia after ischemic stroke. Neurology 2000;54:1124-1131.

3. Gorelick P, Scuteri A, Black S, et al. Vascular contributions to cognitive impairment and dementia: a statement for healthcare professionals from the American Heart Association/American Stroke Association. Stroke 2011;42: 2672-2713.

4. Smith E, Schneider J, Wardlaw J, Greenberg S. Cerebral microinfarcts: the invisible lesions. Lancet Neurol 2012; 11:272-282.

5. Blum S, Luchsinger JA, Manly JJ, et al. Memory after silent stroke: hippocampus and infarcts both matter. Neurology 2012;78:38-46.

6. Schaapsmeerders P, van Uden I, Tuladhar A, et al. Ipsilateral hippocampal atrophy is associated with long-term memory dysfunction after ischemic stroke in young adults. Hum Brain Mapp 2015;36:2432-2442.

7. Wu W, Brickman A, Luchsinger J, et al. The brain in the age of old: the hippocampal formation is targeted differentially by diseases of late life. Ann Neurol 2008;64:698-706.

8. Debette $S$, Seshadri $S$, Beiser A, et al. Midlife vascular risk factor exposure accelerates structural brain aging and $\operatorname{cog}$ nitive decline. Neurology 2011;77:461-468.

9. Jack C, Petersen R, Xu Y, et al. Rates of hippocampal atrophy correlate with change in clinical status in aging and AD. Neurology 2000;55:484-490.

10. Pohjasvaara T, Mäntylä R, Salonen O, et al. MRI correlates of dementia after first clinical ischemic stroke. J Neurol Sci 2000;181:111-117.

11. Brodtmann A, Werden E, Pardoe H, et al. Charting cognitive and volumetric trajectories after stroke: protocol for the Cognition and Neocortical Volume after Stroke (CANVAS) study. Int J Stroke 2014;9:824-828.

12. Adams HP, Bendixen BH, Kappelle LJ, et al. Classification of subtype of acute ischemic stroke: definitions for use in a multicenter clinical trial: TOAST: Trial of Org 10172 in Acute Stroke Treatment. Stroke 1993;24:35-41.

13. Bamford J, Sandercock P, Dennis M, Burn J, Warlow C. Classification and natural history of clinically identifiable subtypes of cerebral infarction. Lancet 1991;337:1521-1526.

14. Brott T, Adams HP, Olinger CP, et al. Measurements of acute cerebral infarction: a clinical examination scale. Stroke 1989;20:864-870.

15. van Swieten JC, Koudstaal PJ, Visser MC, Schouten HJ, van Gijn J. Interobserver agreement for the assessment of handicap in stroke patients. Stroke 1988;19:604-607.

16. Silbert B, Evered L, Scott D, Cowie T. The apolipoprotein $\mathrm{E}$ epsilon4 allele is not associated with cognitive dysfunction in cardiac surgery. Ann Thorac Surg 2008; 86:841-847.

17. Li Q, Pardoe H, Lichter R, et al. Cortical thickness estimation in longitudinal stroke studies: a comparison of 3 measurement methods. Neuroimage Clin 2014;8: 526-535.

18. Boccardi M, Bocchetta M, Ganzola R, et al. Operationalizing protocol differences for EADC-ADNI manual hippocampal segmentation. Alzheimers Dement 2015; 11:184-194.
19. Frisoni G, Jack C, Bocchetta M, et al. The EADC-ADNI Harmonized Protocol for manual hippocampal segmentation on magnetic resonance: evidence of validity. Alzheimers Dement 2015;11:111-125.

20. Woolrich MW, Jbabdi S, Patenaude B, et al. Bayesian analysis of neuroimaging data in FSL. Neuroimage 2009;45:S173-S186.

21. Verardi V, Croux C. Robust regression in Stata. Stata J 2009;9:439-453.

22. Xie M, Yi C, Luo X, et al. Glial gap junctional communication involvement in hippocampal damage after middle cerebral artery occlusion. Ann Neurol 2011;70: 121-132.

23. Gemmell E, Bosomworth H, Allan L, et al. Hippocampal neuronal atrophy and cognitive function in delayed poststroke and aging-related dementias. Stroke 2012;43: 808-814.

24. Fujioka M, Nishio K, Miyamoto S, et al. Hippocampal damage in the human brain after cardiac arrest. Cerebrovasc Dis 2000;10:2-7.

25. Yang YS, Kim SY, Kim J. Ischemic evidence of transient global amnesia: location of the lesion in the hippocampus. J Clin Neurol 2008;4:59-66.

26. Brodtmann A, Pardoe H, Li Q, Lichter R, Ostergaard L, Cumming T. Changes in regional brain volume three months after stroke. J Neurol Sci 2012;322:122-128.

27. Butler TL, Kassed CA, Sanberg PR, Willing AE, Pennypacker KR. Neurodegeneration in the rat hippocampus and striatum after middle cerebral artery occlusion. Brain Res 2002;929:252-260.

28. Sachdev PS, Chen X, Joscelyne A, Wen W, Altendorf A, Brodaty $\mathrm{H}$. Hippocampal size and dementia in stroke patients: the Sydney Stroke Study. J Neurol Sci 2007;260: 71-77.

29. Snaphaan L, Rijpkema M, van Uden I, Fernandex G, de Leeuw FK. Reduced medial temporal lobe functionality in stroke patients: a functional magnetic resonance imaging study. Brain 2009;132:1882-1888.

30. Debette S, Beiser A, DeCarli C, et al. Association of MRI markers of vascular brain injury with incident stroke, mild cognitive impairment, dementia, and mortality. Stroke 2010;41:600-606.

31. Weinstein G, Beiser AS, DeCarli C, Au R, Wolf PA, Seshadri S. Brain imaging and cognitive predictors of stroke and Alzheimer's disease in the Framingham Heart Study. Stroke 2013;44:2787-2794.

32. Brundel M, van den Heuvel M, de Bresser J, Kappelle LJ, Biessels G. Cerebral cortical thickness in patients with type 2 diabetes. J Neurol Sci 2010;299:126-130.

33. Jeerakathil T, Wolf PA, Beiser A, et al. Stroke risk profile predicts white matter hyperintensity volume. Stroke 2004; 35:1857-1861.

34. Knecht S, Oelschlager C, Duning T, et al. Atrial fibrillation in stroke-free patients is associated with memory impairment and hippocampal atrophy. Eur Heart J 2008;29:2125-2132.

35. Stefansdottir H, Arnar DO, Aspelund T, et al. Atrial fibrillation is associated with reduced brain volume and cognitive function independent of cerebral infarcts. Stroke 2013;44:1020-1025.

36. Kobayashi A, Iguchi M, Shimizu S, Uchiyama S. Silent cerebral infarcts and cerebral white matter lesions in patients with nonvalvular atrial fibrillation. J Stroke Cerebrovasc Dis 2012;21:310-317. 
37. Veit R, Kullman S, Heni M, et al. Reduced cortical thickness associated with visceral fat and BMI. Neuroimage Clin 2014;6:307-311.

38. King KS, Peshock RM, Rossetti HC, et al. Effect of normal aging versus hypertension, abnormal body mass index, and diabetes mellitus on white matter hyperintensity volume. Stroke 2014;45:255-257.
39. Ajilore O, Narr K, Rosenthal J, et al. Regional cortical gray matter thickness differences associated with type 2 diabetes and major depression. Psychiatry Res Neuroimaging 2010; 184:63-70.

40. Peng B, Chen Z, Ma L, Dai Y. Cerebral alterations of type 2 diabetes mellitus on MRI: a pilot study. Neurosci Lett 2015;606:100-105.

\section{This Week's Neurology ${ }^{\circledR}$ Podcast}

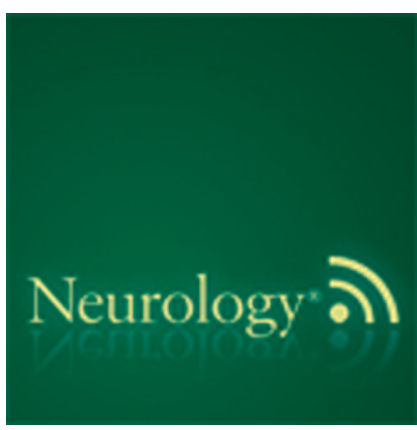

Antiepileptic drug therapy in patients with autoimmune epilepsy (see the July 2017 issue of Neurology ${ }^{\circledR}$ Neuroimmunology \& Neuroinflammation)

This podcast begins and closes with Dr. Robert Gross, Editor-inChief, briefly discussing highlighted articles from the July 11, 2017, issue of Neurology. In the first segment, Dr. Lara Marcuse talks with Dr. Anteneh Feyissa about his paper on antiepileptic drug therapy in patients with autoimmune epilepsy published in Neurology: Neuroimmunology \& Neuroinflammation. In the second part of the podcast, Dr. Stacey Clardy focuses her interview with Dr. Jeffrey A. Cohen on new immunotherapies in neurology.

Disclosures can be found at Neurology.org.

At Neurology.org, click on "RSS" in the Neurology Podcast box to listen to the most recent podcast and subscribe to the RSS feed.

No CME will be offered this week: Interviews based on articles from Neurology ${ }^{\circledR}$ Clinical Practice, Neurology ${ }^{\circledR}$ Genetics, and Neurology: Neuroimmunology \& Neuroinflammation are excluded from the CME program.

\section{Visit the Neurology ${ }^{\circledR}$ Resident \& Fellow Website}

Click on Residents \& Fellows tab at Neurology.org.

Now offering:

- Neurology ${ }^{\circledR}$ Resident \& Fellow Editorial team information

- "Search by subcategory" option

- E-pearl of the Week

- RSS Feeds

- Direct links to Continuum ${ }^{\circledR}$, Career Planning, and AAN Resident \& Fellow pages

- Recently published Resident \& Fellow articles

- Podcast descriptions

由 1 Fincesook Find Neurology ${ }^{\circledR}$ Residents \& Fellows Section on Facebook: http://tinyurl.com/o8ahsys

twitter Follow Neurology ${ }^{\circledR}$ on Twitter: http://twitter.com/GreenJournal 


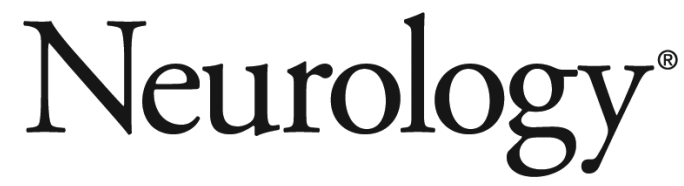

\section{Structural MRI markers of brain aging early after ischemic stroke Emilio Werden, Toby Cumming, Qi Li, et al. \\ Neurology 2017;89;116-124 Published Online before print June 9, 2017 \\ DOI 10.1212/WNL.0000000000004086}

This information is current as of June 9, 2017

Neurology ${ }^{\circledR}$ is the official journal of the American Academy of Neurology. Published continuously since 1951, it is now a weekly with 48 issues per year. Copyright Copyright (C) 2017 The Author(s). Published by Wolters Kluwer Health, Inc. on behalf of the American Academy of Neurology.. All rights reserved. Print ISSN: 0028-3878. Online ISSN: 1526-632X.

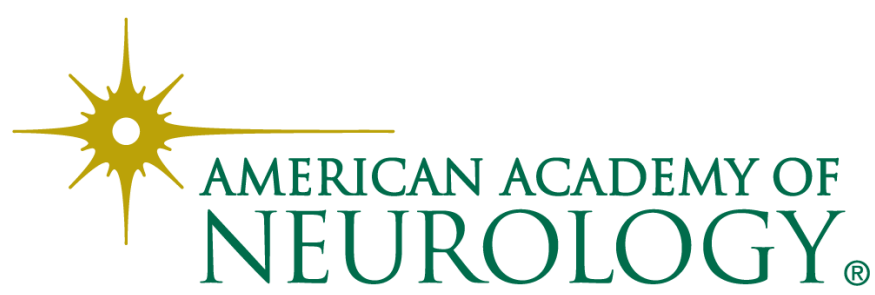




\section{Updated Information \& Services}

\section{Supplementary Material}

\section{References}

Citations

Subspecialty Collections

\section{Permissions \& Licensing}

\section{Reprints}

including high resolution figures, can be found at: http://n.neurology.org/content/89/2/116.full

Supplementary material can be found at: http://n.neurology.org/content/suppl/2017/06/09/WNL.0000000000004 086.DC1

http://n.neurology.org/content/suppl/2017/07/10/WNL.0000000000004 086.DC2

This article cites 40 articles, 14 of which you can access for free at: http://n.neurology.org/content/89/2/116.full\#ref-list-1

This article has been cited by 3 HighWire-hosted articles: http://n.neurology.org/content/89/2/116.full\#\#otherarticles

This article, along with others on similar topics, appears in the following collection(s):

\section{All Cerebrovascular disease/Stroke}

http://n.neurology.org/cgi/collection/all_cerebrovascular_disease_strok e

\section{Alzheimer's disease}

http://n.neurology.org/cgi/collection/alzheimers_disease

Clinical trials Observational study (Cohort, $\overline{\text { Case control) }}$

http://n.neurology.org/cgi/collection/clinical_trials_observational_stud y_cohort_case_control

\section{MRI}

http://n.neurology.org/cgi/collection/mri

Vascular dementia

http://n.neurology.org/cgi/collection/vascular_dementia

Information about reproducing this article in parts (figures,tables) or in its entirety can be found online at:

http://www.neurology.org/about/about_the_journal\#permissions

Information about ordering reprints can be found online:

http://n.neurology.org/subscribers/advertise

Neurology ${ }^{\circledR}$ is the official journal of the American Academy of Neurology. Published continuously since 1951, it is now a weekly with 48 issues per year. Copyright Copyright ( 2017 The Author(s). Published by Wolters Kluwer Health, Inc. on behalf of the American Academy of Neurology.. All rights reserved. Print ISSN: 0028-3878. Online ISSN: 1526-632X.

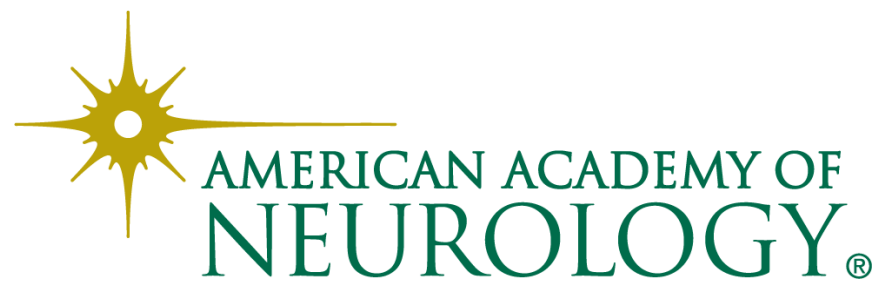

